\title{
Better leukemia-free survival with allogeneic than with autologous HCT in AML patients with isolated trisomy 8: a study from the ALWP of the EBMT
}

\author{
Frédéric Baron $\mathbb{1}^{1} \cdot$ Myriam Labopin $\mathbb{D}^{2,3,4,5} \cdot$ Didier Blaise $\mathbb{D}^{6} \cdot$ Maija Itälä-Remes $^{7} \cdot$ Gérard Socié $\mathbb{D}^{8}$. \\ Edouard Forcade $^{9} \cdot$ Ibrahim Yakoub-Agha $^{10} \cdot$ Norbert Claude Gorin $^{3} \cdot$ Jordi Esteve $^{11} \cdot$ Arnon Nagler $^{2,12}$. \\ Mohamad Mohty $2,3,4,5$
}

Received: 19 June 2020 / Revised: 4 August 2020 / Accepted: 25 August 2020 / Published online: 4 September 2020

(c) The Author(s), under exclusive licence to Springer Nature Limited 2020

\begin{abstract}
The indication for performing an allogeneic hematopoietic stem cell transplantation (allo-HCT) in patients with isolated trisomy $8 \mathrm{AML}$ in first complete remission (CR) is still debated. Here, we compared outcomes of such patients given either allo-HCT or autologous (auto)-HCT. Inclusion criteria consisted of adult patients with de novo AML, isolated trisomy 8, first HCT between 2000 and 2018, CR1 at transplantation, and either auto-HCT or allo-HCT with a HLA-identical sibling donor (MSD) or a 10/10 HLA-matched unrelated donor (UD 10/10). A total of 401 patients met the inclusion criteria. They underwent an auto-HCT $(n=81)$, allo-HCT with a MSD $(n=186)$ or allo-HCT with a 10/10 UD $(n=134)$. At 3 years, relapse incidence, nonrelapse mortality and leukemia-free survival (LFS) were 59\%, 5\%, and 37\%, respectively, in autoHCT recipients; $31 \%(P<0.001), 14 \%(P=0.04)$, and 55\% $(P=0.033)$, respectively, in MSD recipients and $29 \%(P<$ $0.001), 13 \%(P=0.15)$, and $59 \%(P=0.03)$, respectively, in UD 10/10 recipients. In multivariate analysis, in comparison to auto-HCT, MSD and UD 10/10 were associated with a lower risk of relapse $(\mathrm{HR}=0.47, P<0.001$ and $\mathrm{HR}=0.40, P<$ 0.001 , respectively) translating to better LFS (HR $=0.69, P=0.04$ and $\mathrm{HR}=0.60, P=0.03$, respectively). There was also a similar trend for overall survival $(\mathrm{HR}=0.73, P=0.12$ and $\mathrm{HR}=0.65, P=0.08)$.
\end{abstract}

\section{Introduction}

Trisomy 8 is one of the most frequent cytogenetic abnormality in acute myeloid leukemia (AML), occurring in

Supplementary information The online version of this article (https:// doi.org/10.1038/s41409-020-01051-6) contains supplementary material, which is available to authorized users.

Frédéric Baron

f.baron@ulg.ac.be

1 Laboratory of Hematology, GIGA-I3, University of Liege and CHU of Liège, Liege, Belgium

2 EBMT Paris Study Office/CEREST-TC, Paris, France

3 Department of Haematology, Saint Antoine Hospital, Paris, France

4 INSERM UMR 938, Paris, France

5 Sorbonne University, Paris, France

6 Department of Hematology, Institut Paoli Calmettes, Marseille, France
$10-15 \%$ of AML patients and being the sole genetic abnormality in $~ 5 \%$ of AML $[1,2]$. Although allogeneic hematopoietic stem cell transplantation (allo-HCT) has been frequently used in first complete remission (CR) in younger, fit AML patients with isolated trisomy 8 [3, 4], there has been a paucity of data comparing allo-HCT to autologous hematopoietic stem cell transplantation (auto-HCT) or consolidation chemotherapy in these patients [2].

7 Stem Cell Transplantation Unit, HUCH Comprehensive Cancer Center, Helsinki, Finland

8 Hématologie-Greffe AP-HP Hôpital Saint Louis et Inserm U976, Université de Paris, Paris, France

9 Service d'Hématologie Clinique et Thérapie Cellulaire, CHU Bordeaux, F-33000 Bordeaux, France

10 CHU de Lille, LIRIC, INSERM U995, Université de Lille, 59000 Lille, France

11 Hematology Department, Hospital Clínic de Barcelona, Barcelona, Spain

12 Division of Hematology and Bone Marrow Transplantation, Chaim Sheba Medical Center, Tel-Hashomer, Ramat-Gan, Israel 
Specifically, the only large study reported to date assessing the impact of allo-HCT (versus other approaches) included data from 131 patients with trisomy 8 or trisomy 8 plus one additional aberration, and who were treated between 1993 and 2002 in 1 of 8 German AMLSG trials [5]. Among them, 72 patients were eligible for post-remission therapy and received either high-dose cytarabine $(n=43)$, auto-HCT $(n=10)$, or allo-HCT $(n=19$, including 14 with a HLAmatched sibling donor (MSD) and 5 with an unrelated donor (UD)). In multivariate analysis, allo-HCT was associated with better leukemia-free survival (LFS) but was not a prognostic factor for overall survival (OS).

It is now well established that the curative power of alloHCT relies largely on immune-meditated graft-versusleukemia effects [6-8]. Prior studies have observed that CD34+ cells from patients with myelodysplastic syndrome harboring trisomy 8 are more sensitive to Fas-mediated apoptosis [9]. One could argue that this might increase their susceptibility to graft-versus-leukemia effects [10]. In contrast, AML blasts from isolated trisomy 8 patients were shown to exhibit lower expression of several proapoptotic genes than AML blasts from AML patients with a normal karyotype [11], perhaps limiting their susceptibility to be killed by donor immune cells.

Based on these considerations, in the current study we elected to compare transplantation outcomes of AML patients with isolated trisomy 8 in first $\mathrm{CR}$ who underwent auto-HCT versus allo-HCT from either a MSD or a 10/10 HLA-matched unrelated (UD 10/10) donor.

\section{Patients and methods}

\section{Inclusion criteria}

This is a retrospective study from the acute leukemia working party (ALWP) of the European Society for Blood and Marrow Transplantation (EBMT). The EBMT registry is a voluntary working society of more than 600 transplant centers, participants of which are required once a year to report all consecutive HCT and follow-up. Audits are routinely performed to check for data accuracy.

Inclusion criteria included adult patients (defined as $\geq 18$ years of age at transplantation), de novo AML, isolated trisomy 8, first HCT between 2000 and 2018, CR1 at transplantation, and either auto-HCT or allo-HCT with either a MSD or an UD 10/10.

\section{Definitions}

Reduced intensity conditioning (RIC) was defined as regimens combining fludarabine with either $<6 \mathrm{~Gy}$ total body irradiation (TBI), $\leq 8 \mathrm{mg} / \mathrm{kg}$ busulfan, or $\leq 140 \mathrm{mg} / \mathrm{m}^{2}$ melphalan, or with other nonmyeloablative drugs as previously reported $[12,13]$. Acute and chronic graft-versushost disease (GVHD) was graded according to previously reported criteria [14].

\section{Statistical analyses}

Analyses were carried out on data from all patients meeting the inclusion/exclusion criteria. Start time was the day of either auto-HCT or allo-HCT for all endpoints. Patients were censored at the time of last follow-up. Relapse was defined as the presence of $5 \%$ bone marrow blasts and/or reappearance of the underlying disease. Nonrelapse mortality was defined as death without evidence of relapse or progression. OS was defined as the time from allo-HCT to death, regardless of the cause. Events in the composite endpoint LFS included relapse and death, whichever occurred first. The Kaplan-Meier method was used to estimate the probabilities of OS and LFS.

Cumulative incidence functions were used to estimate relapse incidence and nonrelapse mortality in a competing risk setting. Relapse and death were treated as competing events for analyses assessing cumulative incidences of acute or chronic GVHD.

Univariate analyses were performed using Gray's test for cumulative incidence functions and the log-rank test for OS and LFS.

Multivariate Cox models were used to adjust the comparison of transplantation outcomes between patients given an auto-HCT versus either an allo-HCT with MSD or an an allo-HCT with a UD 10/10 donor. Factors included in the model consisted of time from diagnosis to transplantation, patient age, and year of transplantation. Further, in order to take into account the heterogeneity in the effect of a characteristic or a treatment across centers, we introduced a random effect (also named frailty effect) in Cox multivariate models [15]. Then, the same random effect was shared by all patients within the same center. All tests were two sided. The type I error rate was fixed at 0.05 for determination of factors associated with time to event outcomes. Statistical analyses were performed with SPSS 19 (SPSS Inc, Chicago, IL), and R 3.4.3 (R Development Core Team, Vienna, Austria) software packages.

\section{Results}

\section{Patients}

Data from 401 patients meeting the inclusion/exclusion criteria of the study were included in the current analysis. Eighty-one received an auto-HCT, and 320 an allo-HCT from either a MSD $(n=186)$ or an UD 10/10 $(n=134)$. 
Age at transplantation and Karnofsky performance status were comparable in the three groups (Table 1). In contrast, median year of transplantation was earlier in auto-HCT patients (2006) than in MSD (2011) or UD 10/10 (2015) patients (global $P<0.001$ ). The stem cell source was peripheral blood stem cells in $94 \%$ of auto-HCT patients, $76 \%$ of MSD patients, and 85\% of UD 10/10 recipients (global $P=0.002)$. The proportion of patients with mutated NPM1 or FLT3-ITD was comparable within the 3 groups. Finally, among allo-HSCT recipients, the proportion of patients given grafts after RIC regimen was $48 \%$ in MSD and $58 \%$ in UD 10/10 patients, respectively.

\section{Engraftment and GVHD}

Graft failure occurred in one auto-HCT (1\%), four MSD (2\%), and two UD 10/10 (1\%) patients, respectively. Median time to achieve 500 neutrophils was 14 days (IQR 12-17 days) in auto-HCT patients, 16 days (IQR 14-20 days) among MSD patients, and 18 (IQR 15-21 days) among UD 10/10 recipients (global $P<0.001$ ).

Among MSD patients, grade II, III and IV acute GVHD was observed in $27(15 \%), 7(4 \%)$, and 5 (3\%) patients, respectively. In UD 10/10 recipients the figures were 29 $(22 \%), 11(8 \%)$, and $5(4 \%)$, respectively. The 2-year cumulative incidence of chronic GVHD was $42 \%$ in MSD recipients and $43 \%$ in UD $10 / 10$ patients.

\section{Relapse and nonrelapse mortality}

The 3-year cumulative incidence of relapse was 59\% $(95 \%$ confidence interval (CI): 46-69\%) in auto-HCT patients, $31 \%$ (95\% CI: $24-38 \%)$ in MSD recipients $(P=0.0002$ in comparison to auto-HCT), and $29 \%$ (95\% CI: $20-38 \%$ ) in UD 10/10 recipients $(P<0.0001$ in comparison to auto-HCT) (Fig. 1). These observations held true in FLT3 wild-type patients, in those with wild-type NPM1 and in those with both FLT3 and NPM1 wild-type (Supplementary Table 1). In addition, these observations held also true in patients transplanted from 2000 to 2009 as well as in those transplanted from 2010 to 2018 (Supplementary Table 2). Further, combining data from MSD and UD 10/10 patients, there was no impact of conditioning intensity on the relapse risk $(P=$ 0.75). In multivariate analysis, in comparison to auto-HCT, allo-HCT with MSD (Hazard ratio $(\mathrm{HR})=0.47,95 \% \mathrm{CI}$ : $0.31-0.72 ; P=0.0006)$ or UD $10 / 10(\mathrm{HR}=0.40,95 \% \mathrm{CI}$ : $0.24-0.67 ; P=0.0005)$ were each associated with a lower risk of relapse (Table 2) while there was no interaction between donor type and year of transplantation and the risk of relapse.

The 3-year cumulative incidence of nonrelapse mortality was $5 \%(95 \%$ CI: $1-12 \%)$ in auto-HCT patients, $14 \%(95 \%$ CI: $9-20 \%)$ in MSD recipients $(\mathrm{P}=0.04$ in comparison to
auto-HCT), and 13\% (95\% CI: $8-20 \%)$ in UD 10/10 recipients $(P=0.15$ in comparison to auto-HCT) (Fig. 1). These observations held true in patients transplanted from 2000 to 2009 as well as in those transplanted from 2010 to 2018 (Supplementary Table 2). Further, combining data from MSD and UD 10/10 patients, there was no impact of conditioning intensity on nonrelapse mortality $(P=0.52)$. In multivariate analysis, in comparison to auto-HCT, alloHCT with MSD was associated with a higher nonrelapse mortality $(\mathrm{HR}=2.67$, 95\% CI: $1.0-6.9 ; P=0.04)$. AlloHCT with UD 10/10 showed a trend toward a higher nonrelapse mortality $(\mathrm{HR}=2.55,95 \% \mathrm{CI}$ : $0.86-7.5 ; P=0.09)$ compared with auto-HCT (Table 2). There was no interaction between donor type and year of transplantation and the risk of nonrelapse mortality.

\section{LFS and OS}

The 3-year LFS was 37\% (95\% CI: $25-48 \%)$ in auto-HCT patients, 55\% (95\% CI: 47-63\%) in MSD recipients $(P=$ 0.03 in comparison to auto-HCT), and 59\% (95\% CI: 49-68\%) in UD $10 / 10$ recipients $(P=0.003$ in comparison to auto-HCT) (Fig. 2). These observations held true in FLT3 wild-type patients, in those with wild-type NPM1 and in those with both FLT3 and NPM1 wild-type (Supplementary Table 1). Further, combining data from MSD and UD 10/10 patients, there was no impact of conditioning intensity on LFS $(P=0.50)$. In multivariate analysis, in comparison to auto-HCT, allo-HCT with $\mathrm{MSD}(\mathrm{HR}=0.69,95 \% \mathrm{CI}$ : $0.48-0.99 ; P=0.044)$ or UD $10 / 10$ (HR $=0.60,95 \% \mathrm{CI}$ : $0.39-0.94 ; P=0.027)$ were each associated with better LFS (Table 2). There was no interaction between donor type and year of transplantation and LFS.

The 3-year OS was 50\% (95\% CI: 38-61\%) in autoHCT patients, 63\% (95\% CI: 55-70\%) in MSD recipients $(P=0.054$ in comparison to auto-HCT), and $69 \%(95 \%$ CI: $60-78 \%)$ in UD $10 / 10$ recipients $(P=0.01$ in comparison to auto-HCT) (Fig. 2). Further, combining data from MSD and UD 10/10 patients, there was no impact of conditioning intensity on $\mathrm{OS}(P=0.48)$. In multivariate analysis, in comparison to auto-HCT, there was no difference between allo-HCT with $\mathrm{MSD}(\mathrm{HR}=0.73,95 \%$ CI: $0.5-1.1 ; P=0.12)$ or with UD $10 / 10(\mathrm{HR}=0.65$, 95\% CI: $0.4-1.1 ; P=0.08$ ), with respect to OS (Table 2). There was no interaction between donor type and year of transplantation and OS.

Among auto-HCT recipients, the main causes of death were leukemia (56\%), infections (19\%), and hemorrhage (9\%). Among MSD recipients, the main causes of death were leukemia $(51 \%)$, infections $(25 \%)$, and GVHD (16\%). Among UD 10/10 recipients, the main causes of death were leukemia (38\%), GVHD (27\%), and infections (22\%). 
Table 1 Patient characteristics.

\begin{tabular}{|c|c|c|c|c|c|}
\hline \multirow[b]{2}{*}{ Variable } & \multirow{2}{*}{$\begin{array}{l}\text { Auto-HCT } \\
(n=81)\end{array}$} & \multicolumn{2}{|l|}{ Allo-HCT } & \multicolumn{2}{|l|}{$P$ value } \\
\hline & & $\begin{array}{l}\text { MSD } \\
(n=186)\end{array}$ & $\begin{array}{l}\text { UD } 10 / 10 \\
(n=134)\end{array}$ & Auto vs MSD & $\begin{array}{l}\text { Auto vs } \\
\text { UD } 10 / 10\end{array}$ \\
\hline $\begin{array}{l}\text { Follow-up (reverse KM, in } \\
\text { months), median (IQR) }\end{array}$ & $72(47-124)$ & $62(20-98)$ & $35(13-57)$ & 0.20 & $<0.001$ \\
\hline $\begin{array}{l}\text { Patient age at transplant } \\
\text { (year), median (Min-Max) }\end{array}$ & $52(20-72)$ & $51(18-74)$ & $54(19-76)$ & 0.36 & 0.56 \\
\hline Patient sex, $n(\%)$ & & & & 0.56 & 0.11 \\
\hline Male & $40(49)$ & $99(53)$ & $81(60)$ & & \\
\hline Female & $41(51)$ & $87(47)$ & $53(40)$ & & \\
\hline Karnofsky score, $n(\%)$ & & & & 0.67 & 1 \\
\hline$\leq 80$ & $1(1)$ & $5(3)$ & $2(2)$ & & \\
\hline$>80$ & $75(99)$ & $157(97)$ & $124(98)$ & & \\
\hline Missing & 5 & 24 & 8 & & \\
\hline $\begin{array}{l}\text { Diagnosis to transplant (in } \\
\text { months), median (IQR) }\end{array}$ & $5.3(4.3-6.5)$ & $4.5(3.8-5.5)$ & $5(3.9-6.3)$ & $<0.001$ & 0.12 \\
\hline $\begin{array}{l}\text { Year of transplantation, } \\
\text { median (range) }\end{array}$ & $\begin{array}{l}2006 \\
(2000-2018)\end{array}$ & $\begin{array}{l}2011 \\
(2000-2018)\end{array}$ & $\begin{array}{l}2015 \\
(2003-2018)\end{array}$ & $<0.001$ & $<0.001$ \\
\hline NPM1 & & & & 0.18 & 0.19 \\
\hline Non-mutated & $35(76)$ & $92(85)$ & $80(85)$ & & \\
\hline Mutated & $11(24)$ & $16(15)$ & $14(15)$ & & \\
\hline Missing & 35 & 78 & 40 & & \\
\hline FLT3-ITD, $n(\%)$ & & & & 0.9 & 0.16 \\
\hline No & $37(77)$ & $87(76)$ & $67(66)$ & & \\
\hline Yes & $11(23)$ & $27(24)$ & $35(34)$ & & \\
\hline Missing & 33 & 72 & 32 & & \\
\hline \multicolumn{6}{|l|}{ Female to male, $n(\%)$} \\
\hline No & $81(100)$ & $145(78)$ & $113(84)$ & & \\
\hline Yes & 0 & $40(22)$ & $21(16)$ & & \\
\hline Missing & 0 & 1 & 0 & & \\
\hline Cell sources, $n(\%)$ & & & & $<0.001$ & 0.06 \\
\hline Bone marrow & $5(6)$ & $44(24)$ & $20(15)$ & & \\
\hline PBSC & $75(94)$ & $142(76)$ & $114(85)$ & & \\
\hline Missing & 1 & 0 & 0 & & \\
\hline \multicolumn{6}{|l|}{ Conditioning, $n(\%)$} \\
\hline $\mathrm{BuCy}$ & $25(36)$ & $53(28)$ & $18(13)$ & & \\
\hline BuFlu & $1(1)$ & $53(28)$ & $63(47)$ & & \\
\hline FluMel & 0 & $18(10)$ & $6(4)$ & & \\
\hline BuMel & $13(19)$ & 0 & 0 & & \\
\hline BuVP16 & $9(13)$ & 0 & 0 & & \\
\hline СуTBI & $4(6)$ & $17(9)$ & $6(4)$ & & \\
\hline FluTBI & 0 & $13(7)$ & $13(10)$ & & \\
\hline Other & $18(26)$ & $32(17)$ & $28(21)$ & & \\
\hline Missing & 11 & 0 & 0 & & \\
\hline \multicolumn{6}{|l|}{ Conditioning type, $n(\%)$} \\
\hline Myeloablative & & $96(52)$ & $56(42)$ & & \\
\hline Reduced intensity [4] & & $90(48)$ & $78(58)$ & & \\
\hline \multicolumn{6}{|l|}{ In vivo TCD, $n(\%)$} \\
\hline No & $81(100)$ & $130(70)$ & $33(25)$ & & \\
\hline Yes & 0 & $55(30)$ & $101(75)$ & & \\
\hline ATG & & 37 & 94 & & \\
\hline Alemtuzumab & & 18 & 7 & & \\
\hline Missing & 0 & 1 & 0 & & \\
\hline
\end{tabular}

HCT hematopoietic stem cell transplantation, Auto-HCT autologous HCT, Allo-HCT allogeneic HCT, MSD HLA-matched sibling donor, UD 10/ 10 HLA-matched unrelated donor, IQR interquartile range, $P B S C$ peripheral blood stem cells, $B u$ busulfan, $C y$, cyclophosphamide, Flu fludarabine, $\mathrm{Mel}$ melphalan, TBI total body irradiation. 
Fig. 1 Relapse and nonrelapse mortality. Cumulative incidence of relapse (a) and of nonrelapse mortality (b) according to the type of transplantation.
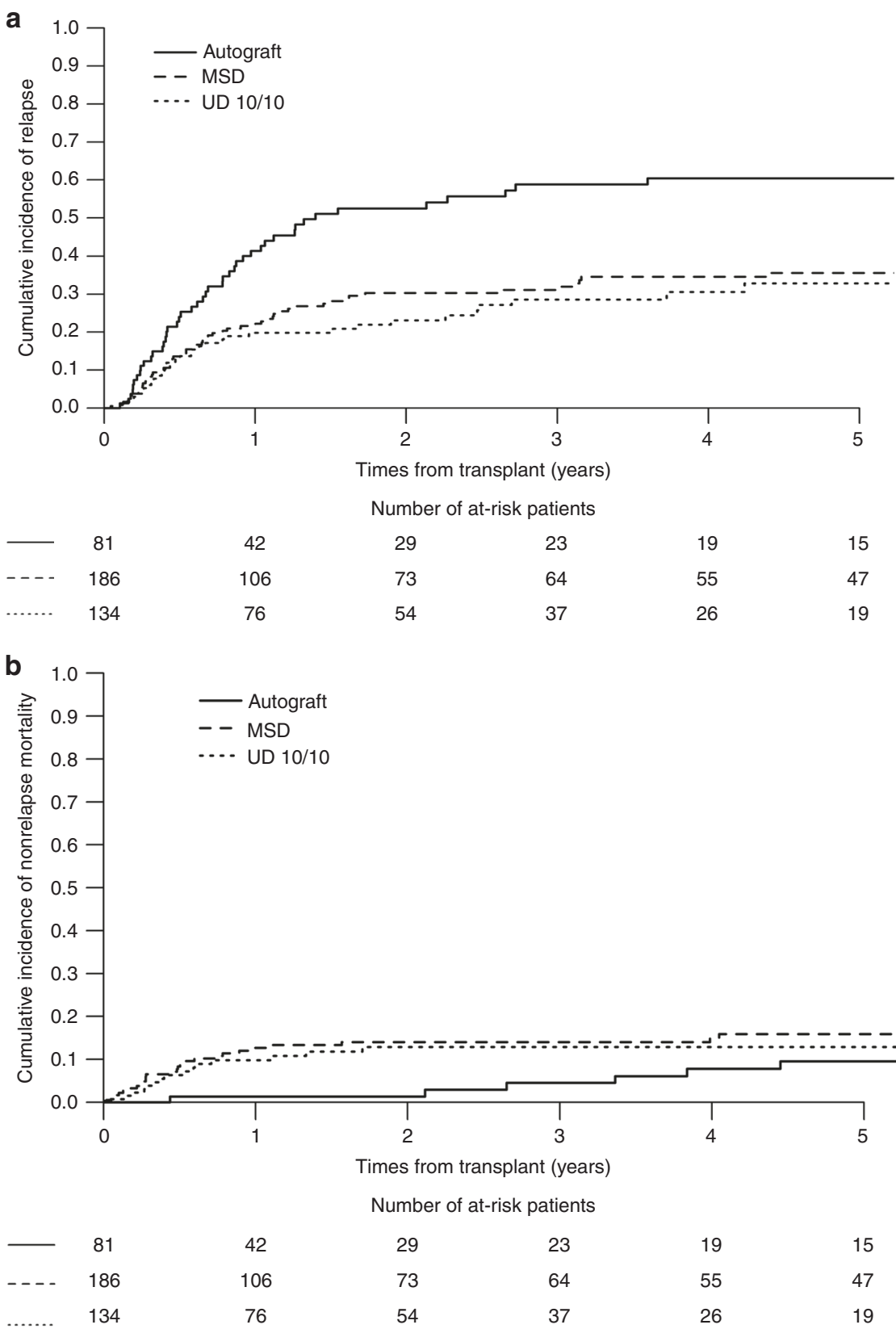

\section{Second transplantation after relapse}

A total of 36 patients received a further allo-HCT after relapse. This include 17 out of 46 relapses $(37 \%)$ in the auto-HCT group, 13 out of 57 relapses $(22.8 \%)$ in the MSD group, and 6 out of 33 relapses (18.2\%) in the UD 10/ 10 group.

\section{Discussion}

Recent studies have demonstrated that AML blasts from patients with trisomy 8 AML have a specific signature marked in part by an overexpression of genes located in chromosome 8 [11]. Interestingly, several genes involved in the apoptosis pathway are downregulated in trisomy $8 \mathrm{AML}$ blasts [11]. Since apoptosis is important for AML blast killing by both chemotherapy and immune cells [16], there is a strong rationale for comparing auto-HCT versus alloHCT specifically in patients with trisomy 8 AML.

Given that patients with isolated trisomy 8 AML are classified in the European LeukemiaNet intermediate-risk group, an allo-HCT for fit AML patients with trisomy 8 in first CR with an appropriate donor has been recommended. However, as mentioned above, this recommendation has not yet been clearly supported by data. In the current study, we 
Table 2 Multivariate analysis of transplantation outcomes.

\begin{tabular}{|c|c|c|c|c|c|c|c|c|}
\hline & \multicolumn{2}{|l|}{ Relapse } & \multicolumn{2}{|l|}{ NRM } & \multicolumn{2}{|l|}{ LFS } & \multicolumn{2}{|l|}{ OS } \\
\hline & HR $(95 \% \mathrm{CI})$ & $P$ value & HR $(95 \% \mathrm{CI})$ & $P$ value & HR $(95 \% \mathrm{CI})$ & $P$ value & $\mathrm{HR}(95 \% \mathrm{CI})$ & $P$ value \\
\hline Auto-HCT (reference) & 1 & & 1 & & 1 & & & \\
\hline Allo-HCT MSD & $0.47(0.31-0.72)$ & 0.0006 & $2.67(1-6.9)$ & 0.043 & $0.69(0.48-0.99)$ & 0.044 & $0.73(0.5-1.1)$ & 0.12 \\
\hline Allo-HCT UD 10/10 & $0.40(0.24-0.67)$ & 0.0005 & $2.55(0.86-7.5)$ & 0.09 & $0.60(0.39-0.94)$ & 0.027 & $0.65(0.4-1.1)$ & 0.08 \\
\hline Time diag to HCT (mo) & $0.96(0.87-1)$ & 0.35 & $0.97(0.83-1.1)$ & 0.74 & $0.96(0.89-1)$ & 0.30 & $0.98(0.9-1.1)$ & 0.60 \\
\hline Patient age (per 10 y.) & $0.95(0.84-1.1)$ & 0.48 & $1.39(1.1-1.8)$ & 0.014 & $1.04(0.93-1.2)$ & 0.49 & $1.09(0.96-1.2)$ & 0.17 \\
\hline Y. of HCT & $1.01(0.97-1)$ & 0.74 & $0.92(0.86-0.99)$ & 0.022 & $0.98(0.95-1)$ & 0.34 & $0.98(0.94-1)$ & 0.25 \\
\hline Center (frailty) & & 0.36 & & 0.26 & & 0.36 & & 0.26 \\
\hline
\end{tabular}

NRM nonrelapse mortality, LFS leukemia-free survival, $O S$ overall survival, $H C T$ hematopoietic stem cell transplantation, Auto-HCT autologous HCT, Allo-HCT allogeneic HCT, MSD HLA-matched sibling donor, UD 10/10 HLA-matched unrelated donor, $Y$ year.

Statistically significant $p$ values are in bold

report on the largest cohort of AML patients with isolated trisomy 8 who underwent either an auto-HCT or an alloHCT in first CR. Our data clearly demonstrate better LFS with allo-HCT than with auto-HCT, due to a significantly lower risk of relapse. This was true irrespective of donor type (MSD or UD 10/10). There was a similar trend for OS that did not reach statistical significance perhaps because of insufficient statistical power for the OS outcome. In the other hand, more efficient salvage strategies could have been possible among auto-HCT patients. This is illustrated by the observation of a higher proportion of patients in the auto-HCT group than in the two allo-HCT groups receiving a further allo-HCT as treatment for AML relapse. Since quality of life is better after auto-HCT than after allo-HCT [17], further studies are needed to define the role of autoHCT in subgroups of trisomy 8 AML patients with a lower risk of relapse (such as those without detectable minimal residual disease (MRD) at HCT) and a high risk of nonrelapse mortality.

Obviously genetic randomization (i.e., HLA-identical sibling availability versus not) [18-21] or true randomization have remained the gold standard for assessing the role of allo-HCT in AML subtypes. However, since such studies are not likely to be performed in isolated trisomy 8 AML, we must rely on alternative methods for assessing the role of allo-HCT in that setting. Comparison of outcomes between auto-HCT and allo-HCT has been shown to produce valid observations. Furthermore, we were reassured by the fact that the three groups were relatively well balanced for the main AML characteristics (except median time from diagnosis to transplantation which was highest in the auto-HCT group, providing a possible bias in favor of auto-HCT). In order to reduce the risk of further bias as much as possible, we restricted the study to patients in first $\mathrm{CR}$ and we excluded patients receiving allo-HCT from alternative donors. Although we did not have MRD data for the patients included in this study, it is unlikely that this introduced a bias in our main observations (lower relapse incidence and better LFS in allo-HCT patients) since patients with detectable MRD at HCT would have been more likely to receive an allo-HCT than an auto-HCT. We however acknowledge that other factors that might have prompted patients to allo-HCT or auto-HCT are missing in the current analyses. These factors include HCT-CI [22] (the data was missing for $53 \%$ of the patients included in the current study) as well as extensive molecular data. Finally, there was no center effect identified in the Cox models for any of the outcomes.

Although trisomy 8 blasts have a specific signature, trisomy 8 AMLs are heterogeneous in terms of presence of additional molecular mutations [2]. Some of these molecular mutations might have a stronger prognostic impact than the trisomy 8 itself and might also influence the susceptibility of AML blasts to graft-versus-leukemia effects. Unfortunately, we do not have the full molecular profile for the patients in this study. However, we performed sensitivity analyses in the subgroup of patients with known NPM1 and FLT3-ITD status. Interestingly, although the results should be taken with some caution given the relatively low patient numbers, we observed that allo-HCT remained associated with lower relapse incidence as well as with better LFS than auto-HCT in the subgroup of patients with wild-type FLT3 status. The number of patients with mutated NPM1 or FLT3-ITD per group was unfortunately too low to allow assessing the impact of auto-HCT versus allo-HCT in these subgroups.

Interestingly, the transplantation outcomes of allo-HCT patients were not statistically impacted by the intensity of the conditioning regimen in univariate analyses. This in in contrast to what has been observed in the BMT-CTN 0901 trial [23]. This might indicate that graft-versus-leukemia effects are more important than conditioning intensity in 
Fig. 2 LFS and OS according to the type of transplantation.
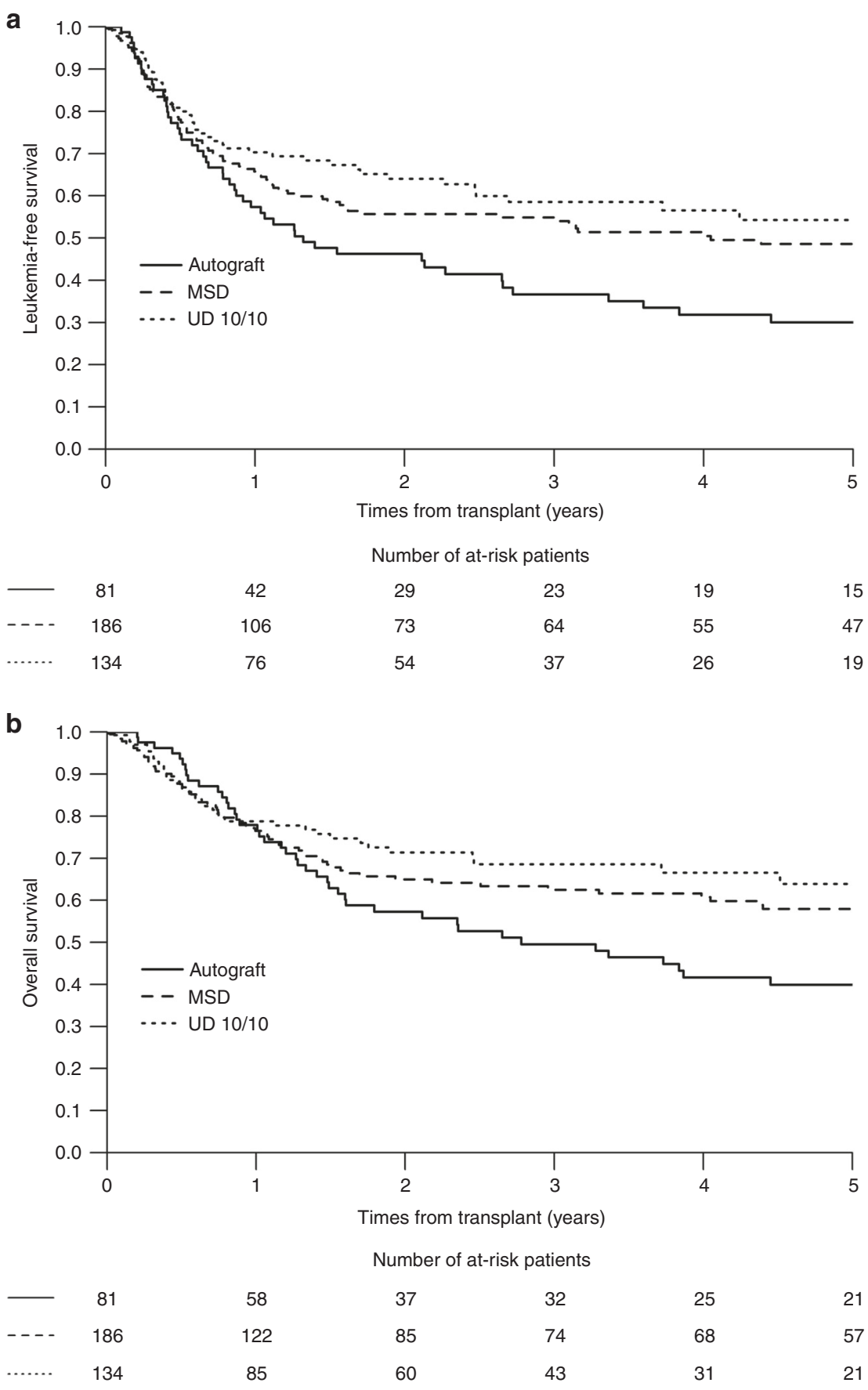

trisomy 8 AML. However, this hypothesis should be taken with extreme caution given the relatively small number of patients (and thus little power to identify risk factors in subgroups) and the lack of data on molecular abnormalities, HCT-CI and on MRD status in a relatively high proportion of patients in the current study, precluding us to build a robust multivariate model assessing the impact of conditioning intensity in allo-HCT patients with trisomy 8 AML. In addition, there was a high heterogeneity in the conditioning regimens in both the RIC and the myeloablative arms with some regimens classified in the RIC group such as the fludarabine plus melphalan one having stronger anti-leukemic activity according to recent studies [24, 25].

In summary, we report here the largest study to date comparing auto-HCT with allo-HCT in AML patients with isolated trisomy 8 in first CR. We observed that allo-HCT in first CR with either a MSD or a UD 10/10 resulted in better LFS than auto-HCT, due to significantly lower risks of disease relapse. 
Acknowledgements We thank Emmanuelle Polge and Audrey Mailhol from the office of the ALWP of the EBMT. FB is Senior Research Associate at the National Fund for Scientific Research (FNRS) Belgium. The list of participating centers is provided in the Supplementary Appendix 1.

Author contributions FB wrote the paper, designed the study, and interpreted the data; ML designed the study, analyzed and interpreted the data, and edited the paper; $\mathrm{AN}$ and $\mathrm{MM}$ designed the study, interpreted the data and edited the paper; DB, MIR, GS, EF, IYA, NCG, and JE reviewed the paper and provided clinical data. All authors approved the final version of the paper.

\section{Compliance with ethical standards}

Conflict of interest FB has received travel grants and/or speaker honoraria from Celgene, AbbVie, Novartis, Pfizer and Sanofi. The other authors declare that they have no relevant conflict of interest in relation with this study.

Ethical approval The scientific board of the ALWP of the EBMT approved this study

Informed consent All patients gave informed consent to participate in retrospective studies

Publisher's note Springer Nature remains neutral with regard to jurisdictional claims in published maps and institutional affiliations.

\section{References}

1. Grimwade D, Walker H, Harrison $G$, Oliver F, Chatters $S$, Harrison C, et al. The predictive value of hierarchical cytogenetic classification in older adults with acute myeloid leukemia (AML); analysis of 10 patients entered into the United Kingdom Medical Research Council AML11 trial. Blood. 2001;98:1312-20.

2. Hemsing AL, Hovland R, Tsykunova G, Reikvam H. Trisomy 8 in acute myeloid leukemia. Expert Rev Hematol. 2019;12:947-58. https://doi.org/10.1080/17474086.2019.1657400

3. Chevallier P, Labopin M, Nagler A, Ljungman P, Verdonck LF, Volin L, et al. Outcome after allogeneic transplantation for adult acute myeloid leukemia patients exhibiting isolated or associated trisomy 8 chromosomal abnormality: a survey on behalf of the ALWP of the EBMT. Bone Marrow Transplant. 2009;44:589-94. https://doi.org/10.1038/bmt.2009.68

4. Konuma $\mathrm{T}$, Kondo $\mathrm{T}$, Yamashita $\mathrm{T}$, Uchida N, Fukuda $\mathrm{T}$, Ozawa $\mathrm{Y}$, et al. Outcome of allogeneic hematopoietic stem cell transplantation in adult patients with acute myeloid leukemia harboring trisomy 8. Ann Hematol. 2017;96:469-78. https://doi. org/10.1007/s00277-016-2909-2

5. Schaich M, Schlenk RF, Al-Ali HK, Dohner H, Ganser A, Heil $\mathrm{G}$, et al. Prognosis of acute myeloid leukemia patients up to 60 years of age exhibiting trisomy 8 within a non-complex karyotype: individual patient data-based meta-analysis of the German Acute Myeloid Leukemia Intergroup. Haematologica. 2007;92:763-70.

6. Weiden PL, Sullivan KM, Flournoy N, Storb R, Thomas ED.and the Seattle Marrow Transplant Team Antileukemic effect of chronic graft-versus-host disease. Contribution to improved survival after allogeneic marrow transplantation. N Engl J Med. 1981;304:1529-33.

7. Baron F, Maris MB, Sandmaier BM, Storer BE, Sorror M, Diaconescu R, et al. Graft-versus-tumor effects after allogeneic hematopoietic cell transplantation with nonmyeloablative conditioning. J Clin Oncol. 2005;23:1993-2003.

8. Baron F, Labopin M, Niederwieser D, Vigouroux S, Cornelissen $\mathrm{JJ}$, Malm C, et al. Impact of graft-versus-host disease after reduced-intensity conditioning allogeneic stem cell transplantation for acute myeloid leukemia: a report from the Acute Leukemia Working Party of the European group for blood and marrow transplantation. Leukemia. 2012;26:2462-8.

9. Sloand EM, Kim S, Fuhrer M, Risitano AM, Nakamura R, Maciejewski JP, et al. Fas-mediated apoptosis is important in regulating cell replication and death in trisomy 8 hematopoietic cells but not in cells with other cytogenetic abnormalities. Blood. 2002;100:4427-32.

10. Dickinson AM, Norden J, Li S, Hromadnikova I, Schmid C, Schmetzer $\mathrm{H}$, et al. Graft-versus-leukemia effect following hematopoietic stem cell transplantation for leukemia. Front Immunol. 2017;8:496 https://doi.org/10.3389/fimmu.2017.00496

11. Virtaneva K, Wright FA, Tanner SM, Yuan B, Lemon WJ, Caligiuri MA, et al. Expression profiling reveals fundamental biological differences in acute myeloid leukemia with isolated trisomy 8 and normal cytogenetics. Proc Natl Acad Sci USA. 2001;98:1124-9. https://doi.org/10.1073/pnas.98.3.1124

12. Baron F, Ruggeri A, Beohou E, Labopin M, Mohty M, Blaise D, et al. Single- or double-unit UCBT following RIC in adults with AL: a report from Eurocord, the ALWP and the CTIWP of the EBMT. J Hematol Oncol. 2017;10:128 https://doi.org/10.1186/ s13045-017-0497-9

13. Sengsayadeth S, Gatwood KS, Boumendil A, Labopin M, Finke J, Ganser A, et al. Conditioning intensity in secondary AML with prior myelodysplastic syndrome/myeloproliferative disorders: an EBMT ALWP study. Blood Adv. 2018;2:2127-35. https://doi. org/10.1182/bloodadvances.2018019976

14. Glucksberg H, Storb R, Fefer A, Buckner CD, Neiman PE, Clift RA, et al. Clinical manifestations of graft-versus-host disease in human recipients of marrow from HL-A-matched sibling donors. Transplantation. 1974; 18:295-304.

15. Andersen PK, Klein JP, Zhang MJ. Testing for centre effects in multi-centre survival studies: a Monte Carlo comparison of fixed and random effects tests. Stat Med. 1999;18:1489-1500.

16. Chang YJ, Zhao XY, Huang XJ. Strategies for enhancing and preserving anti-leukemia effects without aggravating graft-versushost disease. Front Immunol. 2018;9:3041 https://doi.org/10. 3389/fimmu.2018.03041

17. Zittoun R, Suciu S, Watson M, Solbu G, Muus P, Mandelli F, et al. Quality of life in patients with acute myelogenous leukemia in prolonged first complete remission after bone marrow transplantation (allogeneic or autologous) or chemotherapy: a crosssectional study of the EORTC-GIMEMA AML 8A trial. Bone Marrow Transplant. 1997;20:307-15.

18. Suciu S, Mandelli F, de Witte T, Zittoun R, Gallo E, Labar B, et al. Allogeneic compared with autologous stem cell transplantation in the treatment of patients younger than 46 years with acute myeloid leukemia (AML) in first complete remission (CR1): an intention-to-treat analysis of the EORTC/GIMEMAAML-10 trial. Blood. 2003;102:1232-40.

19. Cornelissen JJ, van Putten WL, Verdonck LF, Theobald M, Jacky E, Daenen SM, et al. Results of a HOVON/SAKK donor versus no-donor analysis of myeloablative HLA-identical sibling stem cell transplantation in first remission acute myeloid leukemia in young and middle-aged adults: benefits for whom? Blood. 2007;109:3658-66.

20. Baron F, Efficace F, Cannella L, Muus P, Trisolini S, Halkes CJM et al. Impact of the type of anthracycline and of stem cell transplantation in younger patients with acute myeloid leukaemia: longterm follow up of a phase III study. Am J Hematol. 2020;95:749-58. https://doi.org/10.1002/ajh.25795 
21. Baron F, Efficace F, Cannella L, Willemze R, Vignetti M, Muus P, et al. Long-term follow-up of a trial comparing post-remission treatment with autologous or allogeneic bone marrow transplantation or intensive chemotherapy in younger acute myeloid leukemia patients. Haematologica. 2020;105:e13-e16. https://doi.org/10.3324/ haematol.2019.221333

22. Sorror ML, Maris MB, Storb R, Baron F, Sandmaier BM, Maloney DG, et al. Hematopoietic cell transplantation (HCT)specific comorbidity index: a new tool for risk assessment before allogeneic HCT. Blood. 2005;106:2912-9.

23. Scott BL, Pasquini MC, Logan BR, Wu J, Devine SM, Porter DL, et al. Myeloablative versus reduced-intensity hematopoietic cell transplantation for acute myeloid leukemia and myelodysplastic syndromes. J Clin Oncol: Off J Am Soc
Clin Oncol. 2017;35:1154-61. https://doi.org/10.1200/JCO. 2016.70.7091

24. Baron F, Labopin M, Peniket A, Jindra P, Afanasyev B, Sanz MA, et al. Reduced-intensity conditioning with fludarabine and busulfan versus fludarabine and melphalan for patients with acute myeloid leukemia: a report from the Acute Leukemia Working Party of the European Group for Blood and Marrow Transplantation. Cancer. 2015;121:1048-55. https://doi.org/10.1002/cncr.29163. e-pub ahead of print 2014/11/27

25. Eapen M, Brazauskas R, Hemmer M, Perez WS, Steinert P, Horowitz MM, et al. Hematopoietic cell transplant for acute myeloid leukemia and myelodysplastic syndrome: conditioning regimen intensity. Blood Adv. 2018;2:2095-103. https://doi.org/ 10.1182/bloodadvances. 2018021980 